\title{
Study of system dynamics model and control of a high-power LED lighting luminaire
}

\author{
Bin-Juine Huang*, Po-Chien Hsu, Min-Sheng Wu, Chun-Wen Tang \\ Department of Mechanical Engineering, National Taiwan University, Taipei, Taiwan
}

Received 3 May 2006

\begin{abstract}
The purpose of the present study is to design a current control system which is robust to the system dynamics uncertainty and the disturbance of ambient temperature to assure a stable optical output property of LED. The system dynamics model of the LED lighting system was first derived. A $96 \mathrm{~W}$ high-power LED luminaire was designed and built in the present study. The linearly perturbed system dynamics model for the LED luminaire is derived experimentally. The dynamics model of LED lighting system is of a multipleinput-multiple-output (MIMO) system with two inputs (applied voltage and ambient temperature) and two outputs (forward current and heat conducting body temperature). A step response test method was employed to the $96 \mathrm{~W}$ LED luminaire to identify the system dynamics model. It is found that the current model is just a constant gain (resistance) and the disturbance model is of first order, both changing with operating conditions (voltage and ambient temperature). A feedback control system using PI algorithm was designed using the results of the system dynamics model. The control system was implemented on a PIC microprocessor. Experimental results show that the control system can stably and accurately control the LED current to a constant value at the variation of ambient temperature up to $40^{\circ} \mathrm{C}$. The control system is shown to have a robust property with respect to the plant uncertainty and the ambient temperature disturbance.
\end{abstract}

(C) 2007 Elsevier Ltd. All rights reserved.

Keywords: LED lighting; LED lighting control; High-power LED

\section{Introduction}

The luminous efficiency of high-power light-emitting diode (LED) increases dramatically in recent years. This improves the competitiveness of high-power LED relatively to the traditional lighting devices [1]. According to the estimation of Optoelectronics Industry Development Association (OIDA), the cumulative energy savings for utilization of LED lighting in the US alone could amount to 16.6 Quads ( $760 \mathrm{GW}$ ) of electrical energy by 2020 [2].

A high-power LED lighting system consists of a LED lighting module assembled from plural single LED lamps associated with a proper optical design and a heat dissipation device. The key technology of high-power LED lighting in system application side includes heat dissipation and optical control technique.

\footnotetext{
*Corresponding author.

E-mail address: bjhuang@seed.net.tw (B.-J. Huang).
}

Due to low optical energy efficiency of LED, a highpower LED lighting system needs to dissipate heat to the ambient in quantity which is several times of the conventional lighting device. Heat dissipation is thus an important issue in high-power LED lighting technology [3]. Since the optical properties of a high-power LED lighting system such as brightness and chromaticity are affected by the forward current of LED and the temperature of the LED chip, the design of the lighting luminaire including the heat dissipation device thus indirectly affects the final optical property [4]. In order to obtain a good quality in lighting control and performance reliability of LED, the understanding of system dynamics of a LED lighting system is very important. However, the research of this topic is very rare. The present study intends to derive the system dynamics model of high-power LED lighting system. From that, the development of a control system using the results for providing a robust constant-current driving to LED will be carried out. 


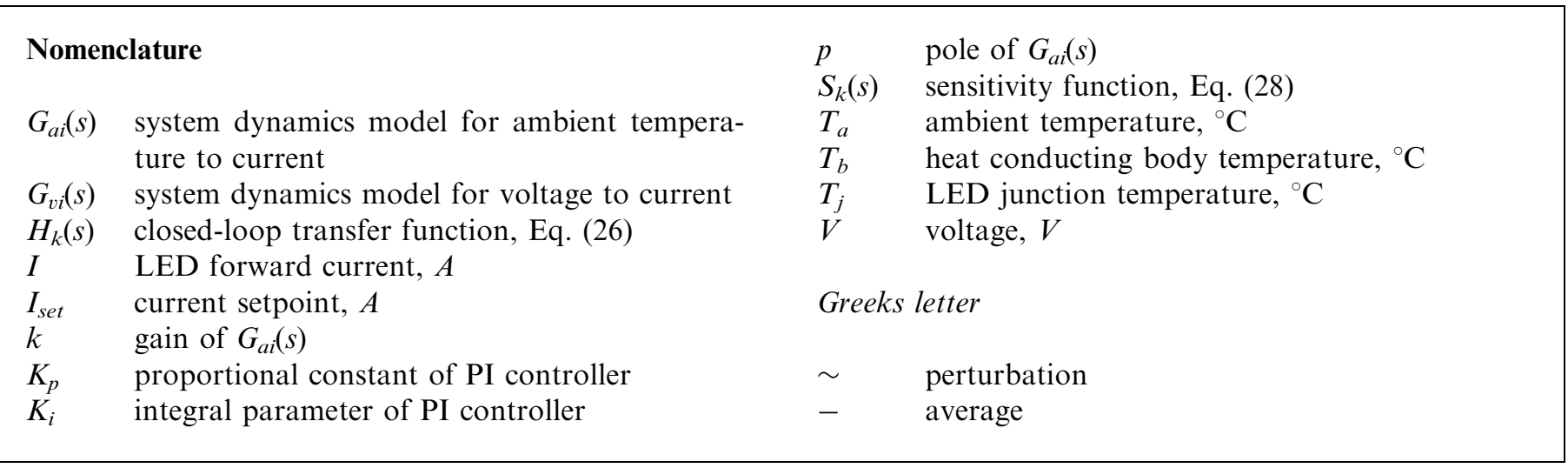

\section{Derivation of system dynamics model of LED lighting system}

\subsection{System dynamics model of a LED lighting luminaire}

The LED lighting system (sometimes called "luminaire") consists of three major components: LED lighting module, heat conducting body, and heat sink. The lighting module includes the light sources (i.e., LED lamps) and secondary optics component. LED lamps are attached to a board for electrical wire connection. The secondary optics component sometimes is added to LED lamps to yield the final optics to the illuminating target. The heat conducting body acts as a thermal connector to the heat sink which is used to dissipate the heat to ambient. The schematic diagram of a LED lighting luminaire is shown in Fig. 1.

For a high-power luminaire, the heat conducting body and the heat sink are usually heavy $(2-10 \mathrm{~kg})$ compared to the LED module. Hence, the thermal response of the whole luminaire is dominated by the connecting body and the heat sink.

Since LED lamp is made from semiconductor, its electrical phenomena is similar to a resistor but with a nonlinear voltage-current relation as shown in Fig. 2.
Thermal phenomena also exist in a LED luminaire due to the effect of energy conversion and heat dissipation process which must obey the law of energy conservation. The system dynamics of a LED luminaire thus can be treated as a multiple-input-multiple-output (MIMO) system with two inputs (applied voltage $V$ and ambient temperature $T_{a}$ ) and two outputs (forward current $I$ and body temperature $T_{b}$ ) as shown in Fig. 3, in perturbed variables defined with respect to the steady value [7]. $G(s)$ is the $2 \times 2$ system transfer function defined as

$G(s) \equiv\left[\begin{array}{cc}G_{v b}(s) & G_{a b}(s) \\ G_{v i}(s) & G_{a i}(s)\end{array}\right]$

from which the following linear-perturbed system relation in Laplace transform holds:

$$
\left[\begin{array}{c}
\tilde{T}_{b}(s) \\
\tilde{I}(s)
\end{array}\right]=\left[\begin{array}{cc}
G_{v b}(s) & G_{a b}(s) \\
G_{v i}(s) & G_{a i}(s)
\end{array}\right]\left[\begin{array}{c}
\tilde{V}(s) \\
\tilde{T}_{a}(s)
\end{array}\right],
$$

where the perturbed variables in time domain are defined as follows:

$$
\tilde{V}(t)=V(t)-\bar{V}
$$

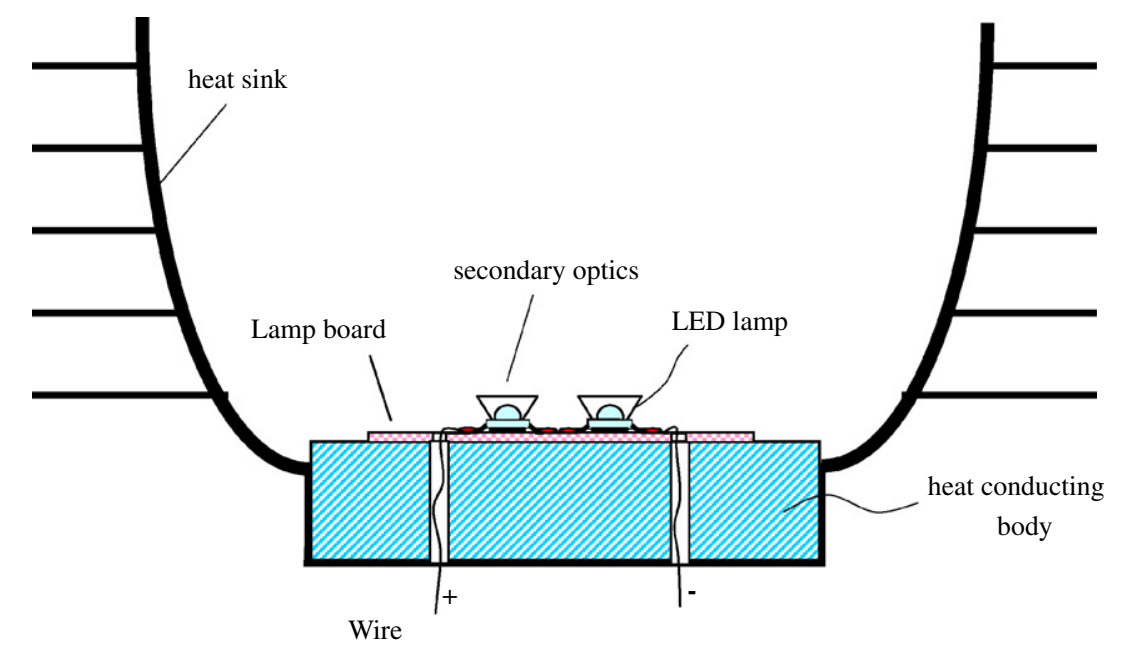

Fig. 1. Configuration of a high-power LED luminaire. 


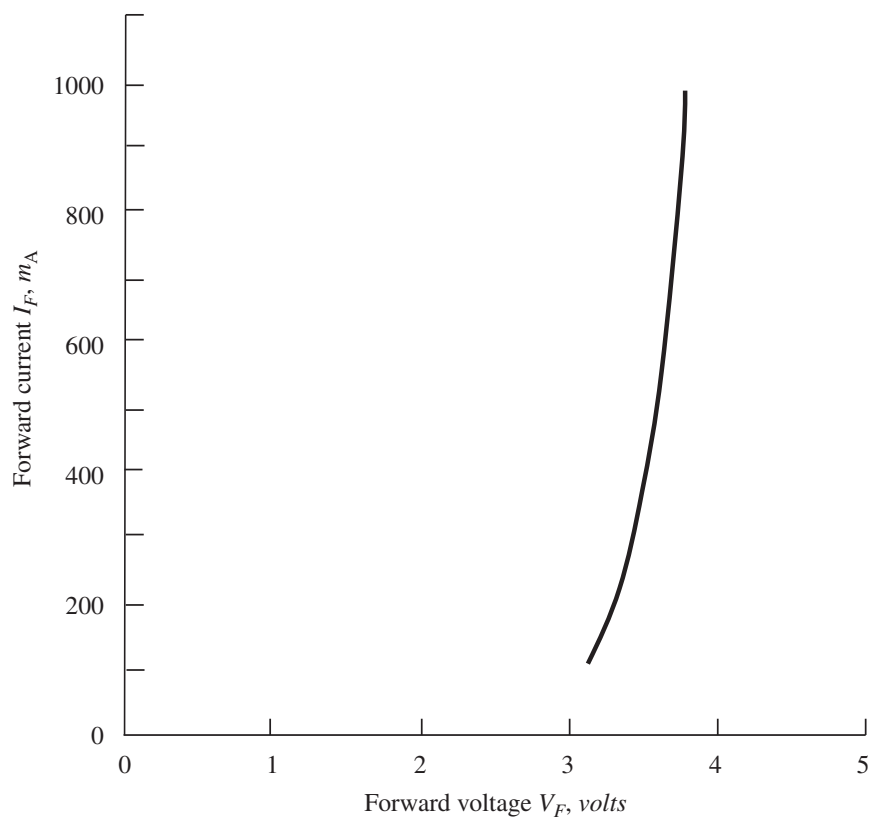

Fig. 2. Typical $I-V$ curve of a LED.

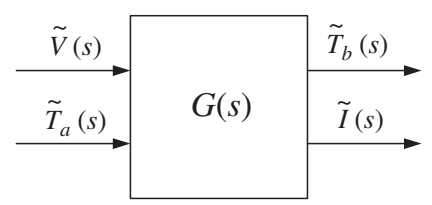

Fig. 3. Input-output block diagram of LED luminaire.

$\tilde{T}_{a}(t)=T_{d}(t)-\bar{T}_{a}$,

$\tilde{T}_{b}(t)=T_{b}(t)-\bar{T}_{b}$,

$\tilde{I}(t)=I(t)-\bar{I}$.

Eq. (2) indicates that both $T_{b}$ and $I$ are affected by ambient temperature $T_{a}$ and applied voltage $V$ which can be written as, in transfer-function form:

$\tilde{T}_{b}(s)=G_{v b}(s) \times \tilde{V}(s)+G_{a b}(s) \times \tilde{T}_{a}(s)$,

$\tilde{I}(s)=G_{v i}(s) \times \tilde{V}(s)+G_{a i}(s) \times \tilde{T}_{a}(s)$.

Fig. 4 shows the MIMO system block diagram.

\subsection{Simplified system dynamics model of LED lighting luminaire}

In order to study the system dynamics model, a $96 \mathrm{~W}$ LED lighting luminaire was designed and fabricated in the present study. The combination of LED lighting module and the heat conducting body is called the light engine. The LED lighting module is made of LED lamps, lamp-holding board and secondary optics component. The luminaire uses two $48 \mathrm{~W}$ lighting engines which are attached to a heat sink made of loop heat pipes (LHP) $[5,6]$. The light engine and the heat sink forms a LED lighting luminaire. Fig. 5 shows

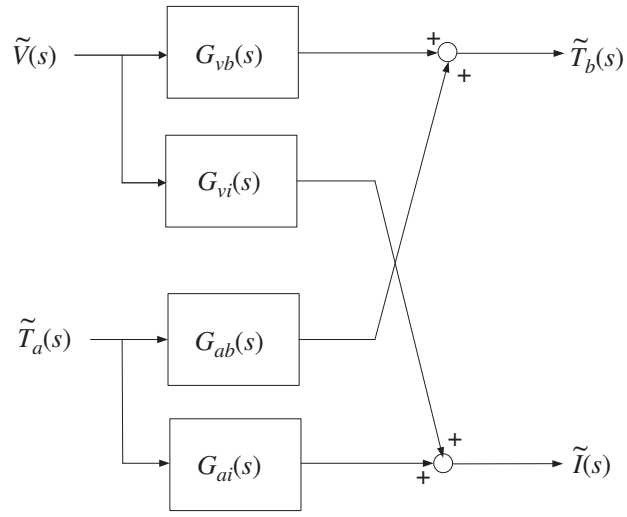

Fig. 4. MIMO block diagram of a LED luminaire.

the detailed dimensions and outlook of the luminaire. The specification of $48 \mathrm{~W}$ LED light engine is shown in Table 1. Luxeon III emitter LXHL-PW09 was used [8]. The total luminaire illumination is around $1824 \mathrm{~lm}$ for $96 \mathrm{~W}$ input, i.e., $19 \mathrm{~lm} / \mathrm{W}$ for net luminous flux output. The total mass of the $96 \mathrm{~W}$ LED luminaire is $4.87 \mathrm{~kg}$.

The LED lighting luminaire is a $2 \times 2$ system with two inputs $\left(V\right.$ and $T_{a}$ ) and two outputs $\left(I\right.$ and $T_{b}$ ). Four dynamics models, $G_{v b}(s), G_{v i}(s), G_{a b}(s)$, and $G_{a i}(s)$ needs to be identified, as shown in Fig. 4. The method of input isolation was employed to identify the four models one by one. In identifying $G_{v b}(s)$ and $G_{v i}(s)$, the input $T_{a}$ (ambient temperature) is kept at constant with $\tilde{T}_{a}(t)=0$ so that

$\tilde{T}_{b}(s)=G_{v b}(s) \tilde{V}(s) \quad$ at $\tilde{T}_{a}(t)=0$,

$\tilde{I}(s)=G_{v i}(s) \tilde{V}(s) \quad$ at $\tilde{T}_{a}(t)=0$.

In identifying $G_{a b}(s)$ and $G_{a i}(s)$, the input $V$ (voltage) is kept at constant with $\tilde{V}(t)=0$ so that

$\tilde{T}_{b}(s)=G_{a b}(s) \tilde{T}_{a}(s) \quad$ at $\tilde{V}(t)=0$,

$\tilde{I}(s)=G_{a i}(s) \tilde{T}_{a}(s) \quad$ at $\tilde{V}(t)=0$.

The models $G_{v b}(s)$ and $G_{a b}(s)$ are related to the response of heat conducting body temperature $T_{b}$ due to the applied voltage $V$ and the ambient temperature $\left(T_{a}\right)$ change.

The ambient temperature $T_{a}$ will indirectly affect the LED junction temperature as well as the optical properties of output light of the luminaire. The effect of $T_{a}$ variation in the LED forward current control should be kept minimal. Since the control of the LED light engine body temperature $T_{b}$ is not very important, the identification of $G_{v b}(s)$ and $G_{a b}(s)$ are ignored and only $G_{v i}(s)$ and $G_{a i}(s)$ are identified in the present study. The final purpose of the present study is to develop a forward current control system for a high-power LED luminaire with robust feedback property against the disturbance of $T_{a}$. Therefore, the simplified dynamics model as shown in Fig. 6 can be used to describe separately the current model $G_{v i}(s)$ and the ambient temperature disturbance model $G_{a i}(s)$. 

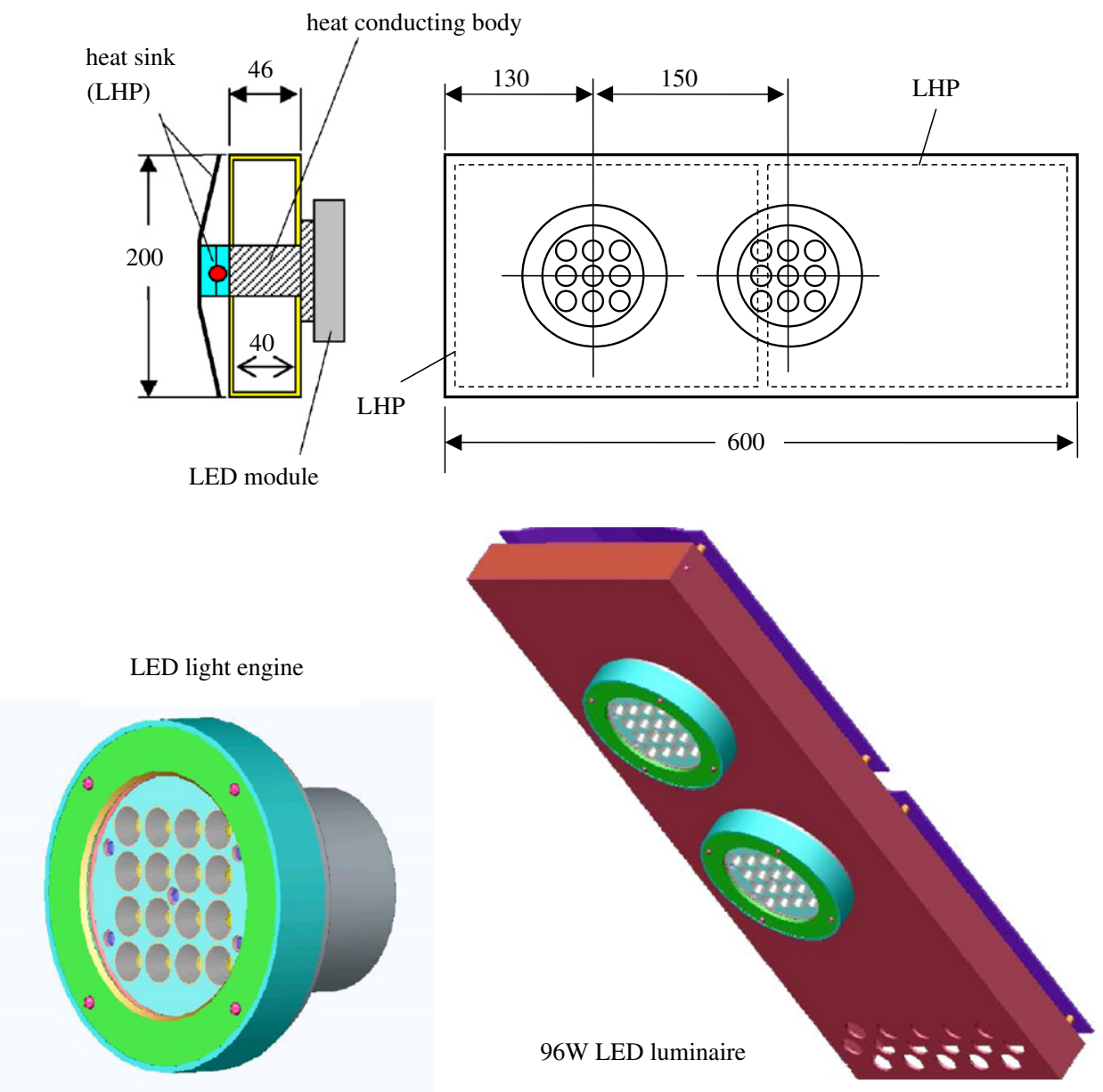

Fig. 5. $96 \mathrm{~W}$ LED luminaire design.

Table 1

Specification of $48 \mathrm{~W}$ LED light engine

Light source (LED lamp)

Luxeon III emitter LXHL-PW09
(80lumen each)
80 lumen@ $900 \mathrm{~mA}, 25^{\circ} \mathrm{C}$ junction (per
lamp)
16
4 by 4
$14 \mathrm{~mm}$
$42 \mathrm{~mm} \times 42 \mathrm{~mm}$, aluminum-based copper
circuit board
$48 \mathrm{~W}$
One set LHP (loop heat pipe) for each
light engine

\subsection{System identification of $96 \mathrm{~W}$ LED lighting luminaire}

The LED forward current is a response of the applied voltage, i.e., the model $G_{v i}(s)=I(s) / V(s)$ represents the current response to the input voltage at constant $T_{a}$.

For LED, the current response $I$ caused by the voltage input $V$ is much faster than $1 \mathrm{~ms}$ and can be approximated as an instantaneous process as compared to the thermal response. Hence, the voltage to current model $G_{v i}(s)$ can be

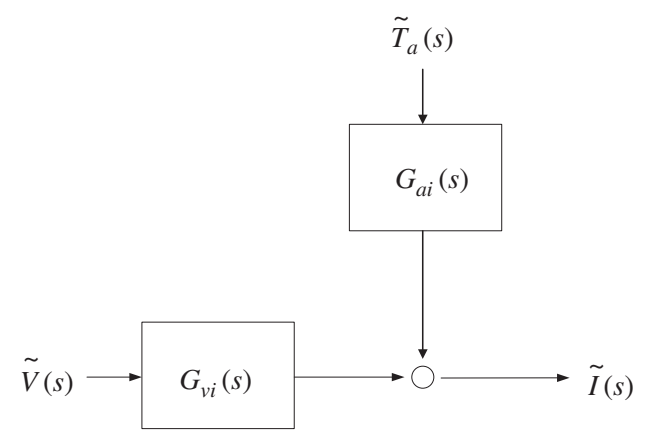

Fig. 6. Simplified model of LED luminaire.

treated as a quasi-steady system with a constant gain. From the current-to-voltage relation of LED as shown in Fig. 2, $G_{v i}(s)$ can be derived from a steady-state test to the LED luminaire.

To determine the model $G_{v i}(s)$, the luminaire is driven by a constant-voltage power supply at a constant ambient temperature. The voltage and current are recorded after a steady state is reached. The results are shown in Fig. 7. Eq. (13) is a current-voltage relation determined from 
experiment at $T_{b}=30 \pm 2{ }^{\circ} \mathrm{C}$.

$I=0.4274 V^{2}-9.082 V+48.29$.

Using quasi-steady assumption for current-voltage response of LED, Eq. (13) can be used to derive a system dynamics model. Substituting the perturbed relations (3) and (6), $V(t)=\bar{V}+\tilde{V}(t)$ and $I(t)=\bar{I}+\tilde{I}(t)$, into Eq. (13) and using the steady-state relation $\bar{I}=0.4274 \bar{V}^{2}-9.082 \bar{V}+48.29$ and neglecting the second-order terms, we obtain a linear perturbation equation:

$\tilde{I}(t)=0.8548 \bar{V} \tilde{V}(t)-9.082 \tilde{V}(t)$.

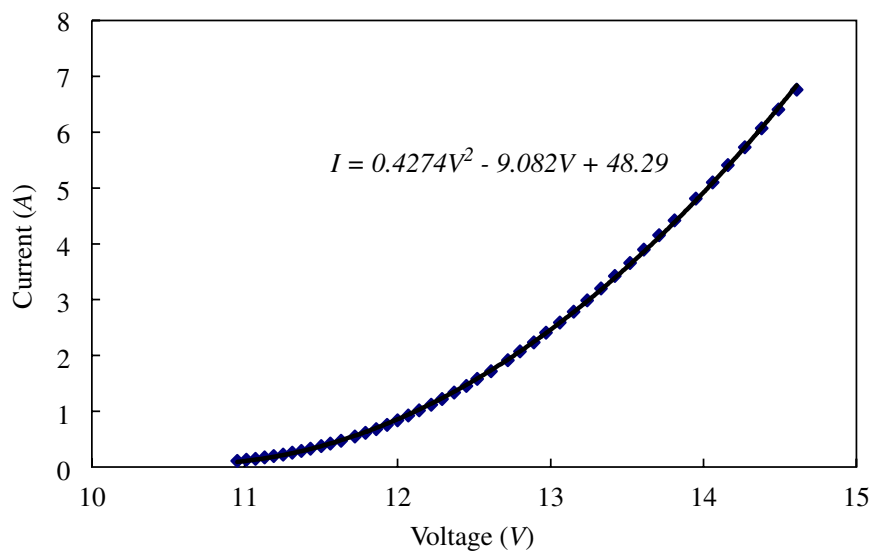

Fig. 7. $I-V$ relation of the $96 \mathrm{~W}$ LED luminaire.
Taking Laplace transfer to Eq. (14), we obtain the dynamics model:

$G_{v i}(s)=\frac{\tilde{I}(s)}{\tilde{V}(s)}=0.8548 \bar{V}-9.082$.

It is seen that $G_{v i}(s)$ varies with operating condition which is represented by $\bar{V}$. For the $96 \mathrm{~W}$ LED luminaire with $4 \times 4$ lamp arrangement, the operating voltage will vary between 11 and $14.5 \mathrm{~V}$. Hence, $G_{v i}(s)$ falls between 0.3206 and 3.312. This means that the system gain of the linear perturbation model could vary about 10 times at different operating conditions. Hence, the robustness of a control system with respect to the large plant variation as shown in the shaded area of Fig. 8 is very important. The average value 2.244 which corresponds to $\bar{V}=13.26 \mathrm{~V}$ can be selected as an average gain for the model $G_{v i}(s)$. Fig. 8 shows that the frequency response of the model $G_{v i}(s)$ at various operating voltage.

To identify the model $G_{a i}(s)$, the step-response test was performed to the $96 \mathrm{~W}$ LED luminaire. T-type thermocouples are used to measure the temperatures of LED heat conducting body and the air inside the chamber. A $0.005 \Omega$ $1 \mathrm{~W}$ resistor is used as the current detector. All the dynamic data are recorded using a YOKOGAWA MV100 recorder with sampling time $2 \mathrm{~s}$. The test facility is shown in Fig. 9. The ambient temperature is simulated using an enclosed chamber with temperature controller. An electric heater is used to heat the chamber using a temperature controller to maintain the chamber in a steady temperature above the room temperature.

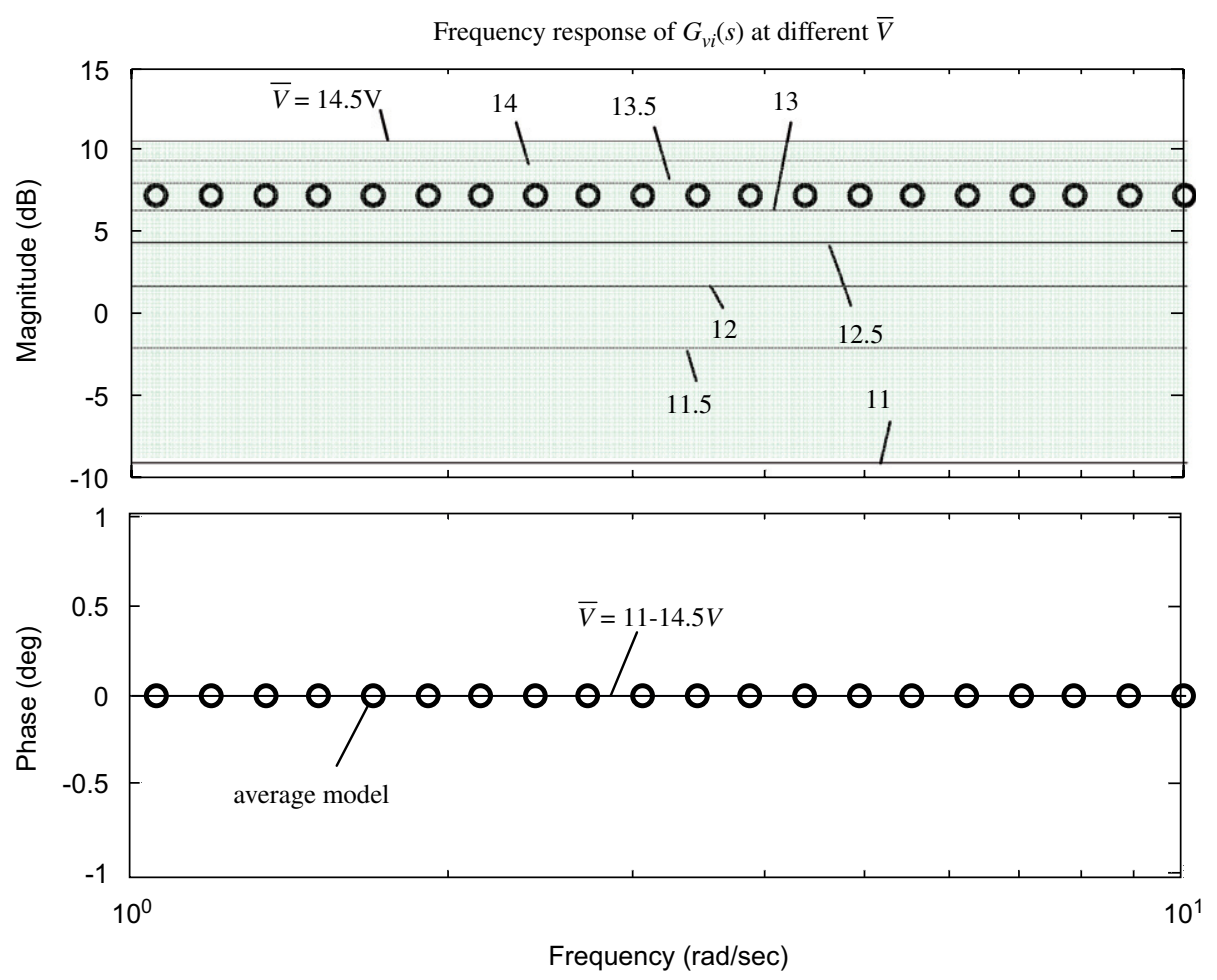

Fig. 8. Frequency response of $G_{v i}(s)$ at different $\bar{V}$. 


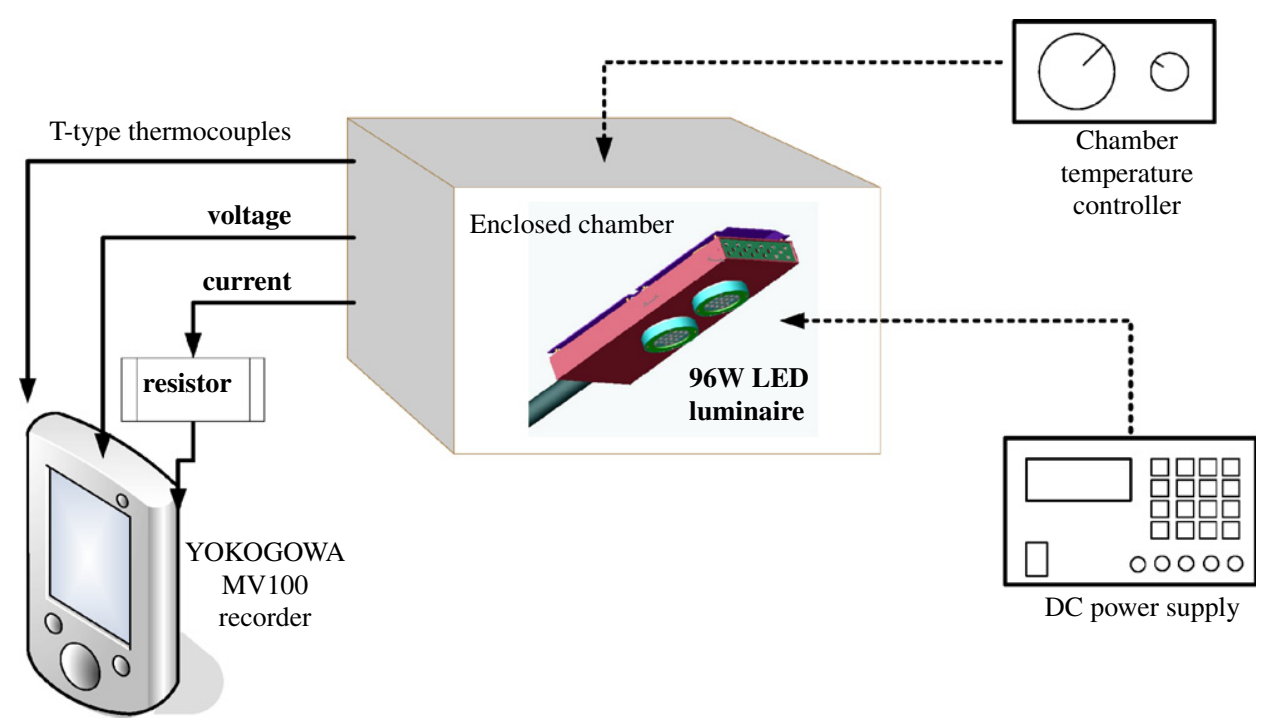

Fig. 9. Test facility setup for step-response test.

By suddenly changing the ambient temperature (by increasing the heating rate to the test chamber) from a steady-state operation and measuring the LED current response, we can obtain the dynamics model from the data analysis.

For a step change of $\tilde{T}_{a}(t)=K u(t), u(t)$ is the unit step function and $\tilde{T}_{a}(s)=K / s$. Taking the time derivative of $\tilde{T}_{a}(t)$, we have

$\tilde{T}_{a}^{\prime}(t) \equiv \frac{\mathrm{d} \tilde{T}_{a}(t)}{\mathrm{d} t}=K \delta(t)$.

Taking the Fourier transform of the time derivative of $\tilde{T}_{a}(t)$, we then obtain

$\tilde{T}_{a}^{\prime}(j \omega) \equiv F\left[\frac{\mathrm{d} \tilde{T}_{a}(t)}{\mathrm{d} t}\right]=j \omega \tilde{T}_{a}(j \omega)=F[K \delta(t)]=K$.

In the meantime, if we take the Fourier transform of the time derivative of the current response $\tilde{I}(t)$, we obtain

$\tilde{I}^{\prime}(\mathrm{j} \omega) \equiv F\left[\frac{\mathrm{d} \tilde{I}(t)}{\mathrm{d} t}\right]=\mathrm{j} \omega \tilde{I}(\mathrm{j} \omega)=F\left[\tilde{I}^{\prime}(t)\right]$.

Combining Eqs. (17) and (18) we obtain

$\frac{\tilde{I}^{\prime}(\mathrm{j} \omega)}{\tilde{T}_{a}^{\prime}(\mathrm{j} \omega)}=\frac{\tilde{I}(\mathrm{j} \omega)}{\tilde{T}_{a}(\mathrm{j} \omega)}=G_{a i}(\mathrm{j} \omega)$.

From Eq. (17), we further obtain

$G_{a i}(\mathrm{j} \omega)=\frac{\tilde{I}^{\prime}(\mathrm{j} \omega)}{K}$.

Eq. (20) depicts that the Fourier transform of the time derivative of the step response function dividing by $K$ is the frequency response of the model $G_{a i}(s)$. Fig. 10 is a typical frequency response of the model $G_{a i}(s)$ at $V=13.85 V$ and $T_{a}$ is imposed a step change from 31.4 to $36.2{ }^{\circ} \mathrm{C}$. The results shown in Fig. 10 indicates that the phase of $G_{a i}(j \omega)$ varies from $0^{\circ}$ at low frequency to approaching $-90^{\circ}$ at high frequency and the gain of $G_{a i}(j \omega)$ has a slope $-20 \mathrm{db} /$ decade. This indicates that the model $G_{a i}(s)$ is probably the first order, Eq. (21).

$G_{a i}(s)=\frac{\tilde{I}(s)}{\tilde{T}_{a}(s)}=\frac{k}{s-p}$.

Fig. 11 is the step response comparison of the measurement with the model prediction using Eq. (21) with $k=0.000684$ and $p=-0.0617$. Fig. 11 also indicates that the current changes $0.1 \mathrm{~A}$ for $5^{\circ} \mathrm{C}$ step change of ambient temperature. This means that the current response due to the ambient temperature variation is not very sensitive for the $96 \mathrm{~W}$ LED luminaire which is relatively heavy compared to a single LED lamp. Table 2 presents the gain $k$ and pole $p$ identified from the step-response test at four operating conditions. The step sizes are all $5^{\circ} \mathrm{C}$. It is seen from Table 2 that the gain $k$ varies from 0.000601 to 0.000709 and the pole $p$ varies from -0.0447 to -0.0617 . Both vary in a narrow range. This implies that the system dynamics model $G_{a i}(s)$ is approximately linear over the operating range. The average value $k=0.000653$ and $p=-0.0484$ can be used to represent an average model. Fig. 12 is the frequency response of $G_{a i}(s)$ at various operating conditions. It is seen that the phases of the four models with parameters shown in Table 2 almost coincide each other. The gain of the various models deviates from the average model by less than $3 \mathrm{db}$.

The above discussions conclude that the system dynamics model of the $96 \mathrm{~W}$ LED luminaire can be approximated by the average model, Eqs. (22) and (23), with uncertainty to some extent.

Current model : $\quad \bar{G}_{v i}(s)=2.244$, 

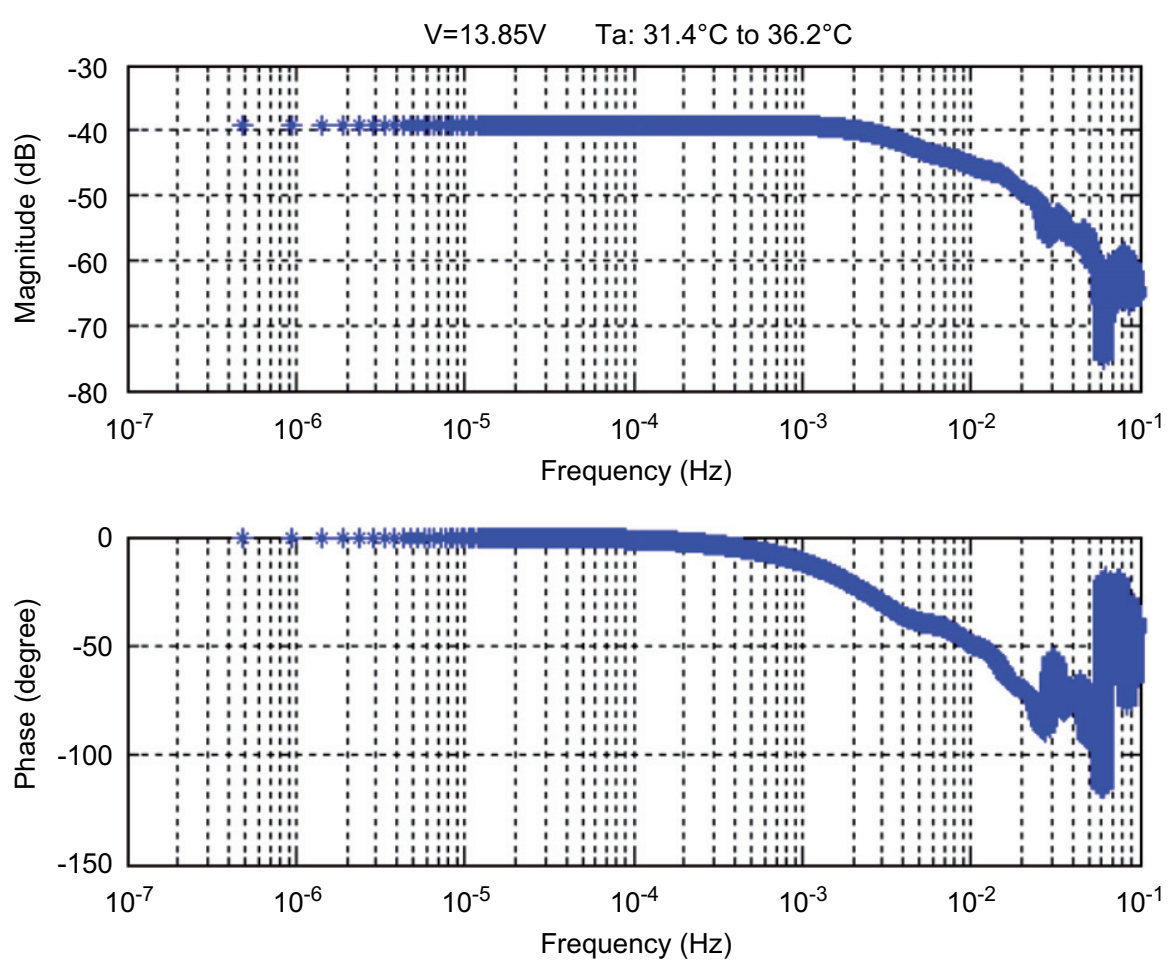

Fig. 10. A typical frequency response of the model.

Table 2

Gains and poles of $G_{a i}(\mathrm{j} \omega)$ identified at different ambient temperature

\begin{tabular}{lllr}
\hline Operating point & Voltage $(\mathrm{V})$ & Step change of ambient temperature $\left({ }^{\circ} \mathrm{C}\right)$ & $k$ \\
\hline 1 & 13.85 & $25.5 \rightarrow 31.4$ & $P$ \\
2 & 13.85 & $31.4 \rightarrow 36.2$ & 0.000709 \\
3 & 13.85 & $36.2 \rightarrow 30.7$ & 0.000684 \\
4 & 13.85 & $30.7 \rightarrow 25.4$ & 0.000624 \\
Average model & & & 0.000601 \\
\hline
\end{tabular}

Ambient temperature disturbance model :

$$
\bar{G}_{a i}(s)=\frac{0.000653}{s+0.0484} \text {. }
$$

\section{Application of system dynamics model in current control}

Steadily controlling the driving input of the LED using a smart control technology to keep the LED in good and steady optical output is very important. A constant-current driver can keep the LED input current at a constant value to assure a relatively steady light output from LED. However, LED is a kind of semiconductor with nonlinear resistance as shown in Fig. 2. This makes the current control not simple. A constant-current power supplier available in the market may not be suitable for all kinds of LED luminaire since the dynamic response of every luminaire may not be all the same.
From the ambient temperature disturbance model $G_{a i}(s)$, Eq. (21), the variation of ambient temperature could cause the current of LED to change even the applied voltage is kept constant. As mentioned previously (Figs. 10 and 11), the response of LED current is not very sensitive to the ambient temperature variation for the $96 \mathrm{~W}$ LED luminaire. The control system will have a good property in ambient temperature disturbance rejection.

From the current model $G_{v i}(s)$, Eq. (15), the current variation due to voltage change falls between 0.3206 and 3.312. This means that the system gain could vary about 10 times at different operating conditions. The feedback system for the current control of LED luminaire as shown in Fig. 13 should be robust with respect to large plant variation.

The average model, Eqs. (22) and (23), can be used as the nominal model for the baseline design of the control system. 


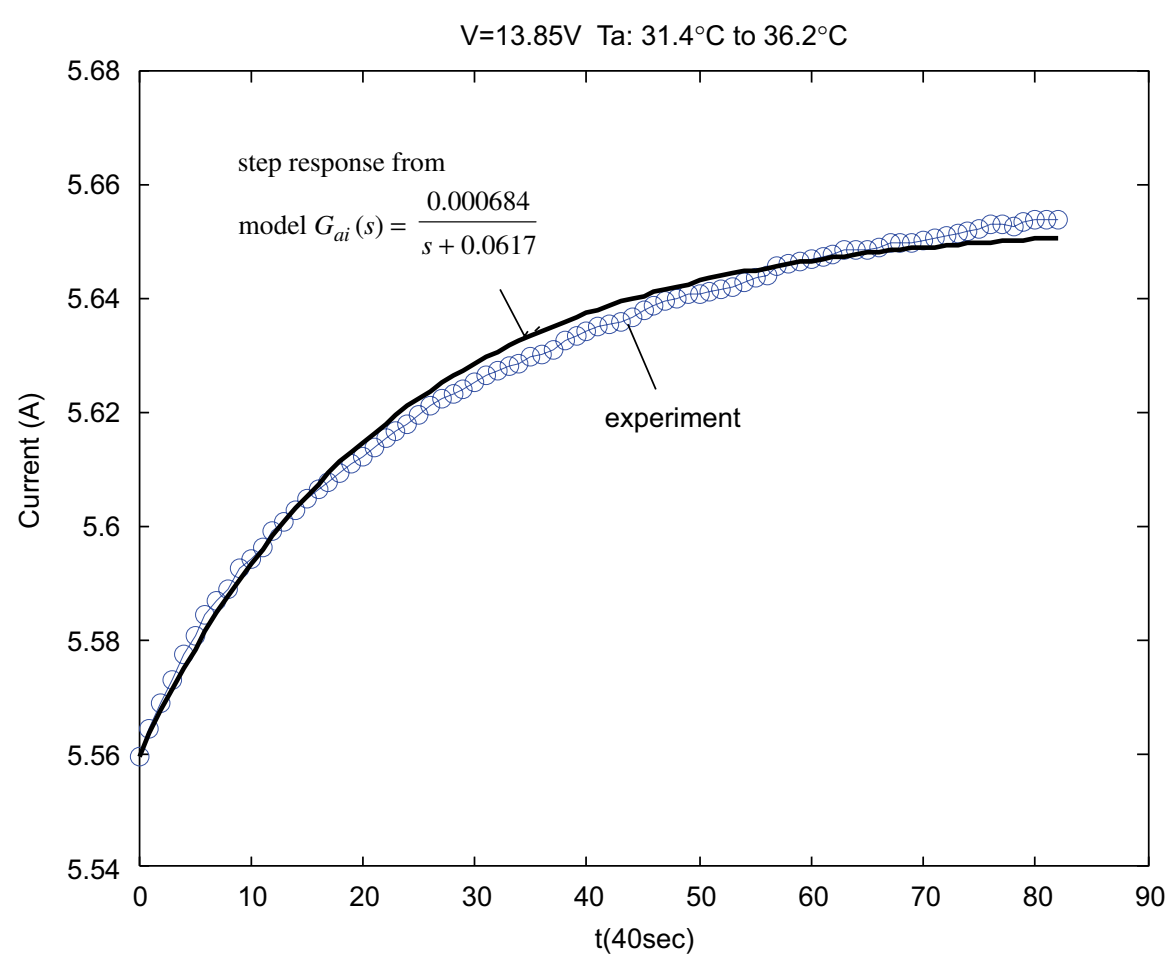

Fig. 11. Step response comparison of experiment and model.
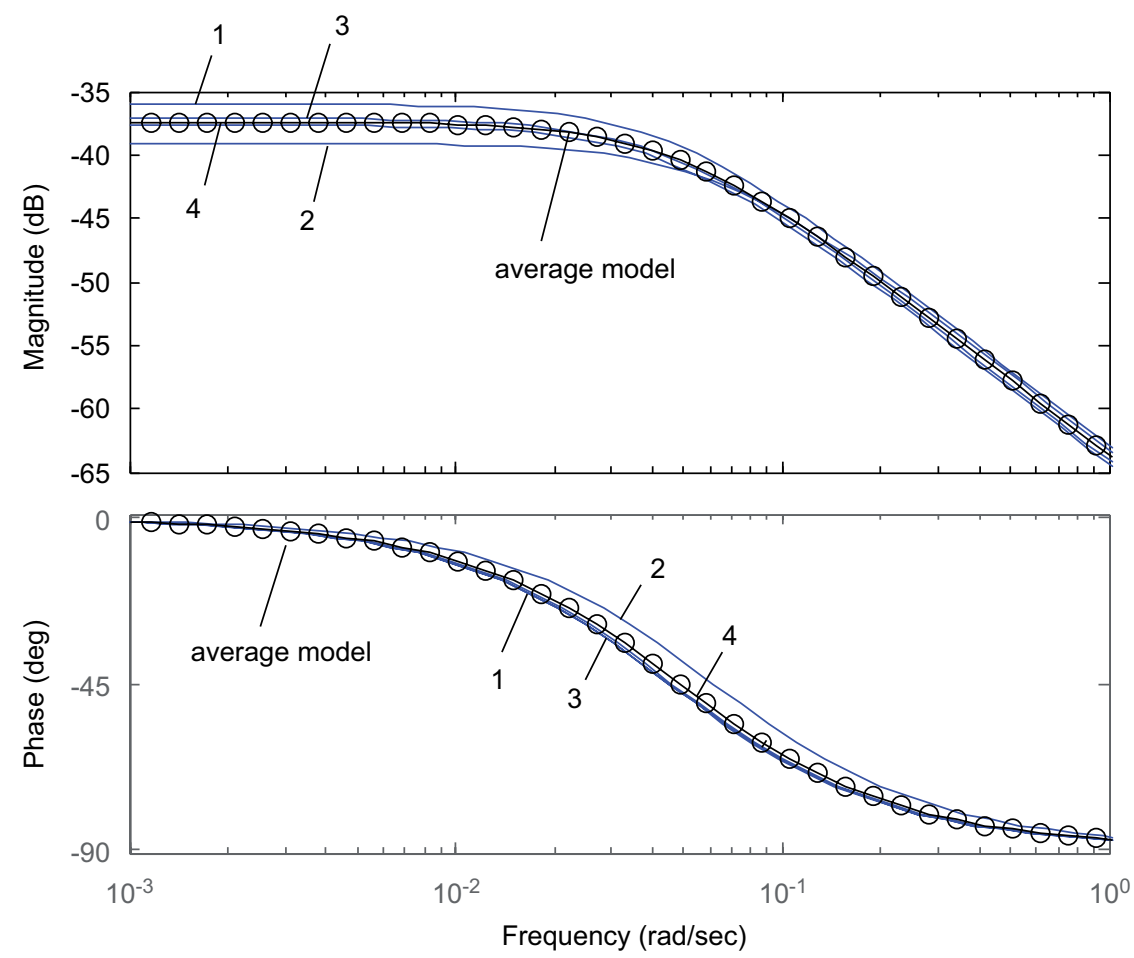

Fig. 12. Frequency response of $G_{a i}(s)$ for $V=13.85 \mathrm{~V}$ at various operating points shown in Table 2 .

\subsection{Feedback control design analysis}

The system dynamics model identified in the present study can be used in the design of the control system. The design of the feedback control system is as shown in
Fig. 13. The controller design analysis can be carried out using the average dynamics model of the $96 \mathrm{~W}$ LED luminaire, Eqs. (22) and (23).

The response of current of the $96 \mathrm{~W}$ LED luminaire with respect to the input voltage change is fast (in zeroth order 


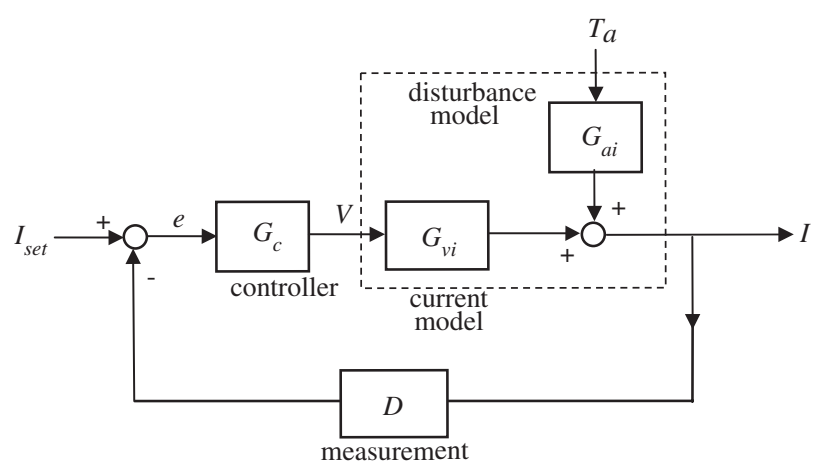

Fig. 13. Feedback control system of LED luminaire.

dynamics). The derivative control is not effective since it is used to suppress the oscillation or overshoot in system response. Proportional-integral (PI) control instead of proportional-integral-derivative (PID) is thus used since the overshoot is not a problem in LED luminaire. Besides, PI control algorism can have robust property with respect to external disturbance if it was carefully tuned $[9,10]$. Eq. (24) is the transfer-function model of the PI controller with proportional constant $K_{p}$ and integral parameter $K_{i}$.

Eq. (25) is the time function of the input voltage to LED luminaire where $e(t) I_{\text {set }}-I(t)$ is the output (current) error.

$$
\begin{aligned}
& G_{c}(s)=K_{p}+\frac{K_{i}}{s}, \\
& V(t)=e(t) K_{p}+K_{i} \int_{0}^{t} e(\tau) \mathrm{d} \tau .
\end{aligned}
$$

PI controller will add a zero and a pole (at origin) to the plant, $G_{v i}(s)$. Properly placing the zero could improve the rise time of the feedback system. PI controller also acts as a low-pass filter which will reject the high-frequency disturbance or noises.

The close-loop transfer function for the set-point (command) response of the feedback system is

$H_{k}(s) \equiv \frac{\tilde{I}(s)}{\tilde{I}_{s e t}(s)}=\frac{G_{c} \bar{G}_{v i}}{1+G_{c} \bar{G}_{v i}}=\frac{2.244 K_{p} s+2.244 K_{i}}{\left(1+2.244 K_{p}\right) s+2.244 K_{i}}$.

From Routh's stability criterion, the PI controller will give a stable closed-loop system if $K_{p}>-0.45$ and $K_{i}>0$. According to the requirement of the feedback system, the PI controller can be further tuned. Since $G_{v i}(s)$ is a constant gain $\left(\bar{G}_{v i}=2.244\right)$ in the baseline design, the closed-loop is first order without overshoot in step response and the steady-state error will be zero. We define the control system requirements of the $96 \mathrm{~W}$ LED luminaire as follows:

(1) rise time (time for step response from $10 \%$ to $90 \%)<1 \mathrm{~s}$;

(2) settling time (time to reach $98 \%$ of steady value) $<6$ s.
Table 3

Tuning of PI controller

\begin{tabular}{llllll}
\hline & Set 1 & Set 2 & Set 3 & Set 4 & Set 5 \\
\hline$K_{p}$ & 1 & 2 & 3 & 4 & 5 \\
$K_{i}$ & 0.8 & 0.9 & 1.1 & 1.2 & 1.3 \\
Rise time (s) & 2.0 & 1.6 & 0.8 & 0.01 & 0.005 \\
Settling time (s) & 4.8 & 5.9 & 5.7 & 5.8 & 5.8 \\
$I A E$ & 2.5907 & 1.7653 & 1.3360 & 1.1242 & 0.5934 \\
\hline
\end{tabular}

We used SIMULINK in MATLAB to tune the parameters of the PI controller $\left(K_{p}\right.$ and $\left.K_{i}\right)$. From the time-domain simulation, the optimal tuning may be carried out by finding the minimum of the integral of the absolute value of the error (IAE) defined in Eq. (27) which also satisfies the above system requirements.

$I A E=\int_{0}^{\infty}|\mathrm{e}(t)| \mathrm{d} t$

The simulation in use with anti-windup for integral control at saturation shows that the effect of $K_{p}$ on control quality is not significant as $K_{p}>5$. For the average model, Eqs. (22) and (23), the resultant feedback property of the control system is listed in Table 3 .

The above results for the PI controller design mainly focus on the set-point response during regulation. As mentioned previously, the PI controller for LED luminaire should be robust with respect to the plant uncertainty. A sensitivity function $S_{k}(s)$ is defined for the closed-loop transfer function with respect to the variation of plant model $G_{v i}(s)$ to describe the effect of the plant uncertainty to the close-loop feedback property:

$S_{k}(s)=\frac{\partial H_{k}}{\partial G_{v i}} \frac{G_{v i}}{H_{k}}=\frac{1}{1+G_{c} G_{v i}}$.

For the average model $\bar{G}_{v i}(=2.244)$ used for baseline design, we obtain

$S_{k}(s)=\frac{s}{\left(1+2.244 K_{p}\right) s+2.244 K_{i}}$.

We used Eqs. (26) and (29) and the parameters listed in Table 3 to further calculate the frequency responses of the closed-loop transfer function $H_{k}(s)$ and the sensitivity function $S_{k}(s)$. Fig. 14 show that all the parameters of PI control listed in Table 3 will result in a low sensitivity to the uncertainty of the plant dynamics model $G_{v i}(\mathrm{~s})$.

The above analysis uses the average model, Eqs. (22) and (23). We repeat the simulation of the control system using different values of the plant model $G_{v i}(\mathrm{~s})$, varying from 0.32 to 3.3. $K_{p}=3$ and $K_{i}=1.1$ are chosen as the acceptable PI controller which satisfies the system requirements and the controller stability margin. 


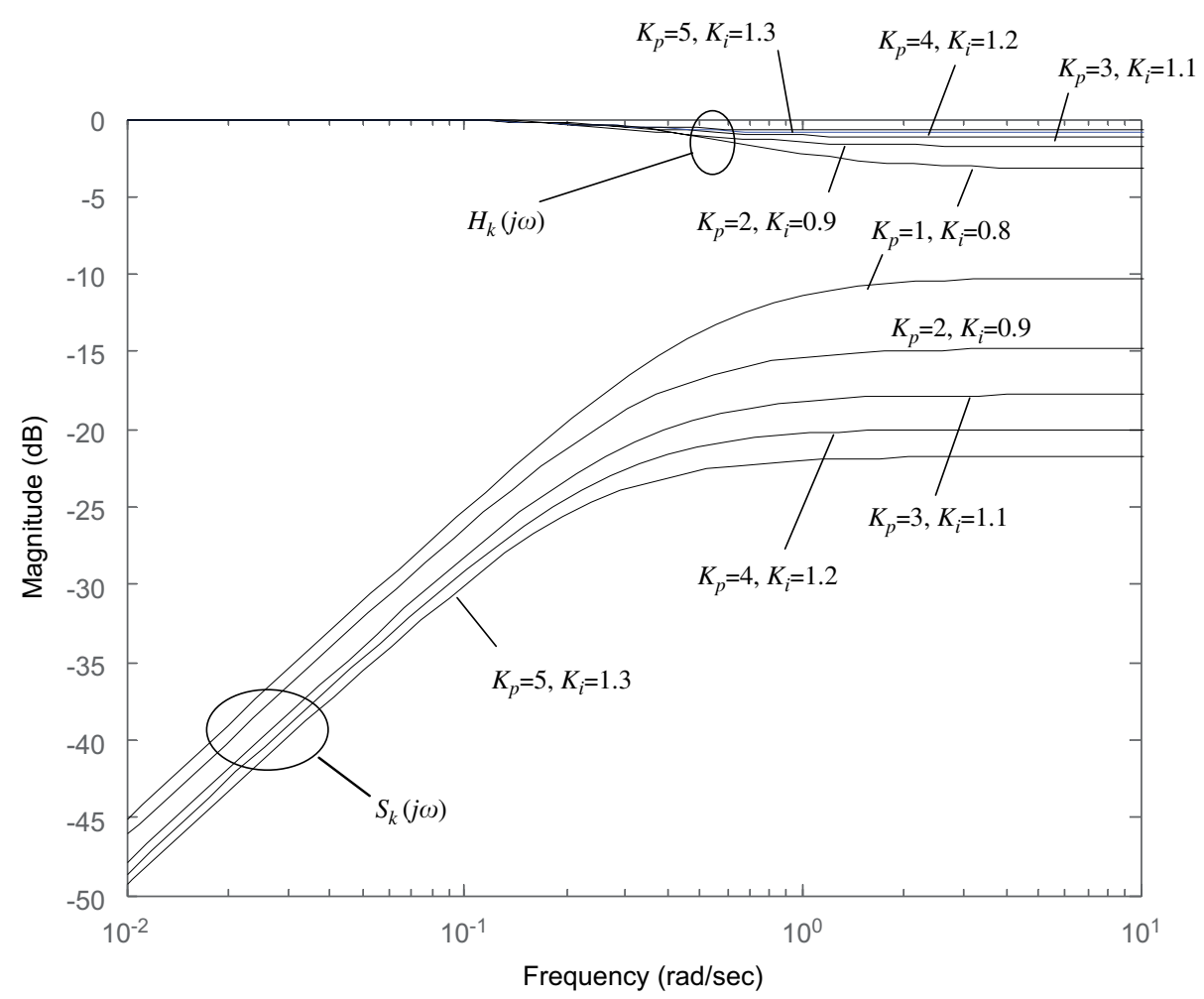

Fig. 14. Frequency response of sensitivity function $S_{k}(s)$.

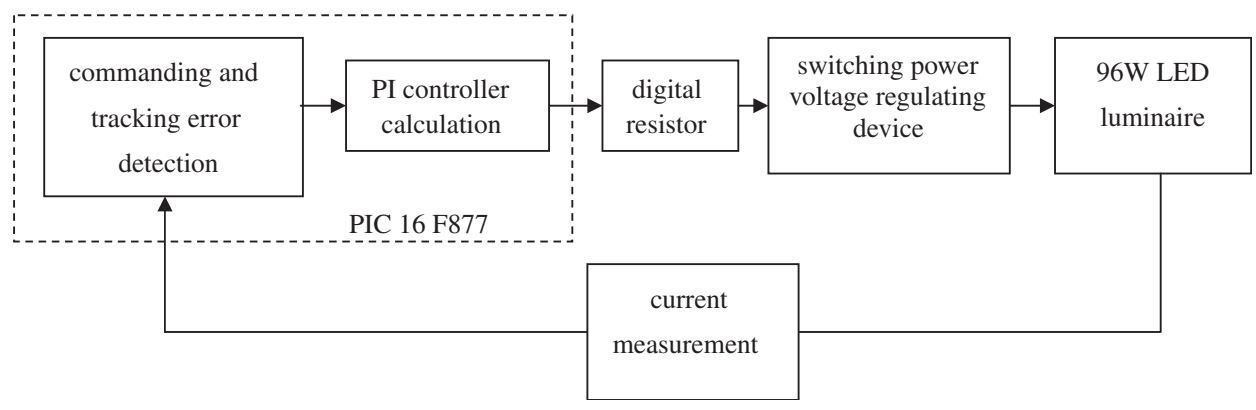

Fig. 15. Control system implementation.

\section{Experimental verification of current control for $96 \mathrm{~W}$ LED luminaire}

The result of the feedback control system design analysis mentioned above is implemented in hardware, as shown in Fig. 15. The control system utilizes the microprocessor PIC 16 F877 as the central processing unit of the digital control system for commanding, error detection and controller calculation. The digital output is sent to a digital resistor (AD8402) which will regulate the voltage to the LED luminaire in a switching power voltage regulating device. The current through LED luminaire is measured using a $5 \mathrm{~m} \Omega$ resistor with an amplifier (IC MAX4373).

Many experiments of the control system were performed at different current setpoints with varying ambient temperature and stagnant air. The results are satisfactory for the current control with robust property. Fig. 16 shows that, for the setpoint $6 \mathrm{~A}$, the current is kept within $6 \pm 0.01 \mathrm{~A}$ all the time, regardless of the ambient temperature variation. Theoretically, the ambient temperature variation could cause LED to change its $I-V$ relation and the system dynamics behavior as well. The control system is shown to have a robust property regarding to the plant uncertainty $G_{v i}(s)$ and the temperature disturbance $G_{a i}(s)$.

Fig. 17 shows the variation of LED junction temperature which is converted from the measured LED base temperature $T_{b}$. It is seen that the junction temperature does not exceed $105^{\circ} \mathrm{C}$ at ambient temperature $40{ }^{\circ} \mathrm{C}$ which is acceptable in application. 


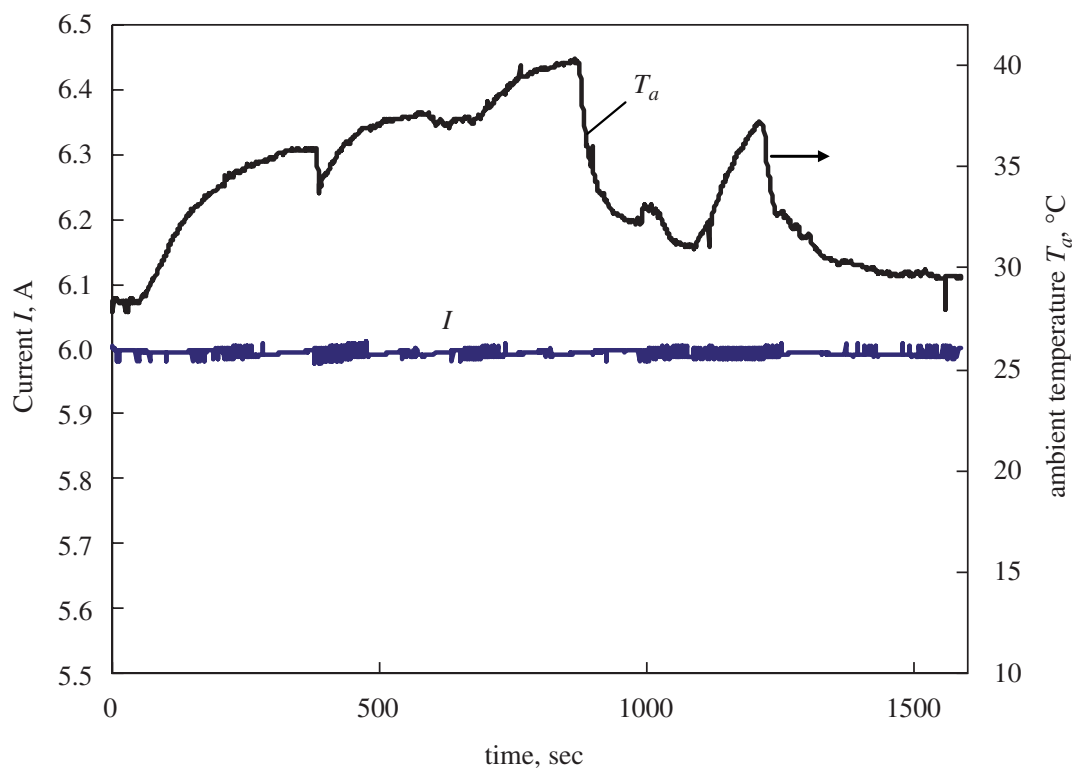

Fig. 16. Current control results at setpoint $I_{\text {set }}=6 \mathrm{~A}$.

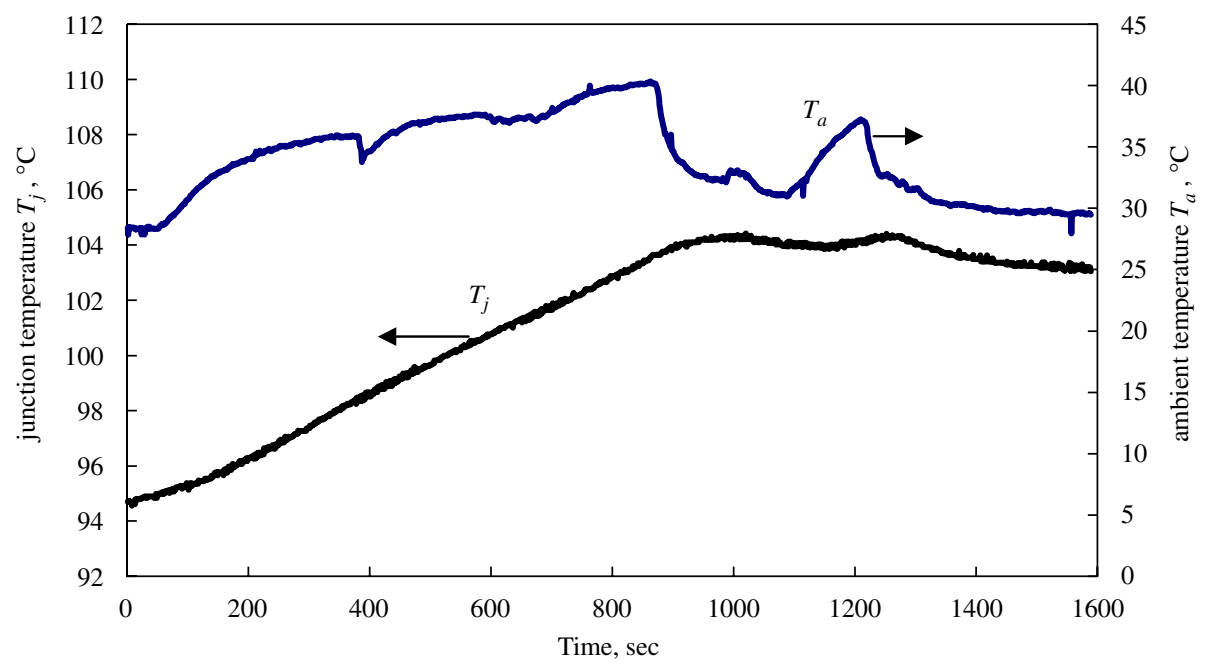

Fig. 17. LED junction temperature variation.

\section{Discussion and conclusion}

The present study intends to derive a system dynamics model of high-power LED lighting system for the development of a robust control system to improve the performance reliability under various operating and environmental conditions. A $96 \mathrm{~W}$ high-power LED luminaire was designed and built for the present study. The linearly perturbed system dynamics model for the LED luminaire is first derived experimentally. The dynamics model of LED lighting system is of a MIMO system with two inputs (applied voltage and ambient temperature) and two outputs (forward current and body temperature). The dynamic behavior can be described separately by the current model and the ambient temperature disturbance model. A step response test method was employed to the $96 \mathrm{~W}$ LED luminaire for the identification of system models. It is found that the current model is just a constant gain (resistance) and the disturbance model is of first order, both changing with operating conditions (voltage and ambient temperature). This means that the dynamics model is subject to uncertainty resulting from operating conditions. The feedback control system thus requires robustness in the presence of disturbance and plant uncertainty.

The system dynamics model $G_{a i}(s)$ identified in the present study indicates that the LED current will change $0.675 \mathrm{~A}$ for a $5^{\circ} \mathrm{C}$ step change of ambient temperature, i.e., steady-state gain is $0.0135 \mathrm{~A} /{ }^{\circ} \mathrm{C}$. This means that the current response due to the ambient temperature variation is not so sensitive for the $96 \mathrm{~W}$ LED luminaire which is relatively heavy compared to a single LED lamp. 
A feedback control system for providing constant-current driving to LED using PI controller was designed and analyzed. An optimal controller was determined using the closed-loop sensitivity function to assure system robustness.

The control system was implemented on a PIC microprocessor. Experimental results show that the control system can stably and accurately control the current to a constant value at the variation of ambient temperature up to $40^{\circ} \mathrm{C}$ and the LED chip junction can be maintained at a temperature lower than $105^{\circ} \mathrm{C}$ at stagnant air condition. It is also shown that the design of luminaire will not affect the control system performance.

The step-response test at four operating conditions indicates that the gain $k$ varies from 0.000601 to 0.000709 and the pole $p$ varies from -0.0447 to -0.0617 . Both vary in a narrow range. This implies that the system dynamics model $G_{a i}(s)$ is approximately linear over the operating range. The average value $k=0.000653$ and $p=-0.0484$ can be used to represent an average model. Since the pole $p$ is inversely proportional to the mass of the LED luminaire. The above average values are for the $96 \mathrm{~W}$ LED luminaire made in the present study. However, we can expect that the steady-state gain of the system dynamics model $G_{a i}(s)$ will increase with decreasing mass of the LED luminaire. If the LED luminaries weight $0.487 \mathrm{~kg}$ (about one tenth of the present LED luminaire), the pole will shift to -0.00484 and the steady-state gain will increase to $1.35 \mathrm{~A} /{ }^{\circ} \mathrm{C}$ (about $22.6 \%$ setpoint). This implies that the sensitivity of the LED current to the ambient temperature change will be very high if the LED luminaire becomes light in weight. The controller design needs to be very careful in treating the disturbance rejection. This situation will probably occur in the indoor LED lighting design with light weight.

\section{Acknowledgment}

The present study was supported by National Science Council, Taiwan and Energy Bureau, Ministry of Economic Affairs, Taiwan.

\section{References}

[1] Tsao JT. Solid-stage lighting: lamps, chips and materials for tomorrow. IEEE Circuits Dev Mag 2004;20(3):28-37.

[2] The promise of Solid State Lighting for general illumination, Optoelectronics Industry Development Association, 2002. Website: http://ighting. sandia.gov/lightingdocs/OIDA_SSL_Roadmap_Summary_2002.pdf $>$.

[3] Shin MW, Thermal design of high-power LED package and system. In: Proceedings of SPIE - the international society for optical engineering, vol 6355. Advanced LEDs for Solid State Lighting, 2006, p. 635509.

[4] Gu Y, Narendran N, Dong T, Wu H. Spectral and luminous efficacy change of high-power LEDs under different dimming methods. In: Proceedings of SPIE - the international society for optical engineering, vol. 6337, sixth international conference on solid state lighting, 2006, p. 63370J.

[5] Huang BJ, Wang TH, Yeh YY, Huang HH, Development of a lowcost LHP for commercial application. In: Thirteenth international heat pipe conference, Shanghai, China, 2004, p. 21-5.

[6] Maydanik YuF. Loop heat pipes. Appl Thermal Eng 2005;25(5\&6): 635-57.

[7] Oppenhen AV, Willsky AS. Signals and system. 2nd ed. Englewood Cliffs, NJ: Prentice-Hall; 1997.

[8] DS45, Luxeon III Emitter Technical Datasheet, Lumileds Lighting; 2006.

[9] Berenguel M, Camacho EF, Garcia-Martin FJ, Rubio FR. Temperature control of a solar furnace. IEEE Control Syst Mag 1999;19(1):8-24.

[10] Åström KJ, Hägglund T. PID controllers. 2nd ed. Research Triangle Park; 1995. 